\title{
The Origin, Development and Winning Skills of Tug of War
}

\author{
Xinyu Li
}

Institute of Physical Education, Yangtze University, Jingzhou 434023, Hubei, China

\begin{abstract}
Tug-of-war is everyone happy of mass sports, which has a long history of the development in the world countries, and now is very popular. This article discusses the origin and development of tug of war. Through the stress distribution analysis of the athletes in process of the tug-of-war, points out that the tug of war victory not only is related to the size of the athletes hard, what is more important, related to the athletes about the direction of the force and skill. According to theoretical analysis of the athletes force and friction, we are more likely to win in the real tug of war.
\end{abstract}

Keywords: Force, friction, mechanical analysis, origin and development, tug-of-war.

\section{INTRODUCTION}

Tug of war is a very common form of mass sports activities, which is widespread in our country, especially in government, schools, troops and other organizations that should have the collective activities. The sport, in England, Ireland, Sweden, Switzerland, Australia, the United States, India, Canada, Italy and other countries, is also a very popular sport [1-3]. Why do people like tug-of-war sport? Because of the simple venues and equipment of tug-of-war competition, especially it is integrated with the fun, competitive, and collective. Tug of war, can improve people's physical fitness, but also enhance the collective sense of honor. Visibly, tug of war, as a kind of temporarily out of work and Labor necessary for survival, and in the spare time for recreation, comfort and joy or their own happiness, it is part of the sports culture, and sports culture influence each other, interaction and mutual promotion. From the perspective of human history and development of human consciousness, to the prosperity of tug-of-war sport is a social and historical development and the inevitable outcome of the development of athletic ability.

\section{THE ORIGIN AND DEVELOPMENT OF TUG OF WAR}

\subsection{The History of Tug of War in China}

In three kingdoms period of Qin and Han dynasties, the war was frequent, so the army strengthened pull-pushed training to improve soldiers fighting capacity. Environmental change in the power of the method and effect of the birth of tug-of-war, produced can be more effective technology to solve practical problems, which can enhance strength and explosive force of soldiers.

The Han dynasty had make the country rich and its military force efficient, folk sports have a bigger development. Putting mental training above physical training was the total trend during Liang-Jin and Northern-Southern dynasties, and sports development was imbalance. At the same time, the Nan dynasty should beat drum with momentum magnificent when tug-of-war started, the momentum of great mass activities had made the ruling class concerns resulting created trouble, Jain Wen emperor Xiao Liang was banned, from now on we could see how the tug-of-war sport development was closely related to the interests of the ruling class, the development of sports culture have direct relationship with the policy of the ruling class $[4,5]$. Among the people because people loved tug-of-war sport, and there was an "anaerobic wins, use to make abundant grains", which was still very popular. In North, because each ethnic group migration, integration more quickly, ruler, and attaching great importance to the practice would choose soldiers, so as to promote the development of martial arts, also contributed to tug of war. Sui dynasty tug-of-war was taking shape, known as the "drama of pull hook".

\subsection{The Origin and Development of Tug-of-War in Other Foreign Countries}

The earliest international tug of war was found at tomb fresco, $2500 \mathrm{BC}$ in Egypt, where had its patterns. Western Europe tug-of-war movement began in $1000 \mathrm{BC}$, which can be read from the Scandinavian and German hero of the story. In the 15th century, the French castle or big villa garden, tug-of-war was a very popular race. Old tug of war, there are many different ways. In Afghanistan, the rope was replaces with a wooden stick to the game, even if no rope, the game could still be performed. Eskimos had called an "Allah" way of one-to-one tug of war competition; both sides were sitting on the ground, through a rope to pull, if pulling each other from sitting position was the determination to win. Late in the development of human history, the tug of war had grown into a sport of physical fitness of sports competitions, not associated with a religious ceremony [6].

The historical record of the tug of war began in the 17th century in English, the game of the England countryside. Up to modern times, the British advocated the modern international tug of war, and the second session of Olympic Games 
in Paris, 1990, the tug of war as a formal event. Then at the third session of the Olympic Games in the United States, at the 4th Olympic Games in Britain and Sweden held in the 5th Olympic Games, tug of war was classified as official event. The 6th Olympic Games not was held for the First World War. The 7th session of the Olympic Games in 1920, given the movement project was various, and tug of war rules failed to be unified, so the international Olympic committee (IOC) in adjusting events cancelled tug of war project, which make it temporarily pause state [7]. Tug-of-war sport of all countries, however, was still in development, Swedish national tug-of-war association was founded in 1933, and then other European countries to emulate, in Britain, the Netherlands 14 countries such as Europe, had formed a tugof-war association. In 1968, the world federation of tug of war was established. It was divided into regional, national, the English premier league, the European Union, world championships and World Cup.

In Japan and South Korea, the tug-of-war originated from China. In December 1980, Japan's national tug-of-war alliance was formally established, according to the international federation of tug-of-war rules as the basis, made the Japanese tug-of-war league indoor game. International federation of tug-of-war indoor and the advantages of the rules of the game, tug of war with Japan alliance proposal in 1982, the international competition was held in the indoor hard floor. in October 1988, Japan held the tug-of-war goodwill assembly with England, a total of eight countries took part in the international conference on goodwill, the international federation of tug-of-war rule revision for indoor outdoor game rules, and in April 1989 to the rules of the international federation of tug of war officially revised to contain interior rules of the game, and in the Netherlands in 1990 held the first session of the world (indoor) tug-of-war championships. Now every two years to organize a tug of war world championship in the world. For centuries, the villagers in South Korea always used tug of war to solve disputes. Surprisingly, 5000 issued by the 1988 Seoul Olympics commemorative coins, there were some engraved with tug of war pattern of the sport. South Korea's popular large tug of war is a very old form of tug of war, and the style of the tug of war originated from Chu dynasty [8]. Today, the fifteenth day of a year, some of the areas in the south of South Korea, is going to have traditional big tug-of-war activities. After the game, the big rope article is divided into tens of thousands of, distribution to contestants and the audience on the spot. South Korea's rope consists of two parts, male, rope and female rope, have pray for good harvest. This custom is not only in Korea, there is also in Japan. In 2004 Japan's Ryukyu Naha city held "super" two-way tug-of-war, billed as the world's largest tug-of-war event, have been listed in the Guinness book of world records. Tug-of-war celebration is also the Ryukyu traditional festival activities, has been 370 years of history.

\section{THE MEANING OF TUG-OF-WAR SPORT}

From military tactics into folk entertainment, Tug-of-war sport transforms into the composition of folk culture in the process. Through the factors of human activities it gives human factors for the natural state, all the natural things is created by human or object is the lasting appeal of culture, that is a cultural power led people to solve these problems. In addition, tug-of-war sport as closely related to people's life phenomenon has become the necessary of human civilization. This kind of sports culture meet the needs of the people to pursue the spiritual life, has penetrated into people's life style, give more and more culture to daily life value and significance.

Tug-of-war sport easily promote its vitality, through the tug of war realize human self-transcendence and the desire to win success, improve the fight ability, realize the value of life, enjoy life. Tug-of-war sport will of individual life value and significance lies in participation, realize the value of individual life, and promote harmony and social harmony. Therefore, we should vigorously advocate and actively carry out tug of war, and promote the construction of spiritual civilization, and based on different dialectical relationship between diversity and unity of the development of human culture, show the general trend of the development of human culture and the basic law.

\section{THE FORCE SITUATION OF THE TUG OF WAR}

The tug of war game develops so widespread throughout the world, and then what factors do we win the tug of war game? Let's analyze the force of the tug of war competition athletes, you will find its factors to win.

\subsection{Physical Model of the Tug of War Rope}

Quality is $\mathrm{m}$ for a piece of rope, it respectively gets pulling force $F_{1}$ and $F_{2}$ along the rope outward on both, and $F_{1}>$ $\mathrm{F}_{2}$, how is the rope tension? To simplify the problem easy to study, rope can be evenly divided into $n$ equal mass, and the quality has focused on the point of the paragraphs, as shown in Fig. (1).

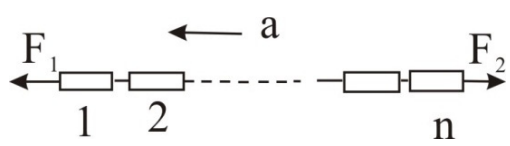

Fig. (1). The force situation of the tug of war rope.

Using the whole method to establish dynamic equation

$\mathrm{F}_{1}-\mathrm{F}_{2}=\mathrm{n}\left(\frac{1}{n} m\right) \mathrm{a}=\mathrm{ma}$

We can obtain

$a=\frac{F_{1}-F_{2}}{m}$

The tension of rope between paragraphs can be obtained by applying the method of isolation, the 1 st and the $2 \mathrm{nd}$, the 2nd and the 3 th, and the (n-1) with the $n$, whose tension is respectively

$T_{1}=\frac{(n-1) F_{1}+F_{2}}{n}=F_{1}-\frac{F_{1}-F_{2}}{n}$

$T_{2}=\frac{(n-2) F_{1}+2 F_{2}}{n}=F_{1}-\frac{2\left(F_{1}-F_{2}\right)}{n}$

$T_{n-1}=\frac{F_{1}+(n-1) F_{2}}{n}=F_{1}-\frac{(n-1)\left(F_{1}-F_{2}\right)}{n}$ 
The above results show that $\mathrm{n}$ is positive integer, $\mathrm{T}_{1}>\mathrm{T}_{2}$ $>\cdots \cdots T_{n-1}$. When the direction to the $F_{1}$ for accelerated motion, in all parts of the rope tension are no equal. The tension of the front is big, the smaller tension of rope back is, and the more the quality of the rope, the greater the acceleration, the difference is obvious. When the rope remains at rest or of uniform motion, the acceleration $\mathrm{a}=0$, then

$$
F_{1}-T_{1}=T_{1}-T_{2}=\cdots \cdots=T_{n-2}-T_{n-1}=T_{n-1}-F_{2}=\frac{m}{n} a=0
$$

And then

$F_{1}=T_{1}=T_{2}=\cdots \cdots=T_{n-1}=F_{2}$

Each point has the same tension. When $\mathrm{a} \neq 0$, and the quality can be left out of the rope timing, $\mathrm{m} \approx 0, \mathrm{~F}_{1}-\mathrm{F}_{2}=\mathrm{m} \approx 0$, also have $F_{1}=T_{1}=\cdots \cdots=T_{n-1}=F_{2}$, rope tension is equal at every point at this time. So when the acceleration a is small, the quality of the rope is also small, rope each point has the same tension, that is $\mathrm{F}_{1}=\mathrm{T}_{1}=\cdots \cdots=\mathrm{T}_{\mathrm{n}-1}=\mathrm{F}_{2}$.

\subsection{Physical Model of the Tug of War Athletes}

In the tug-of-war, athletes are applying pull from each other, so both parties were by the tensile strength of the other athletes, that is to say, both players is applying object, is also a mechanical object, they force analysis is shown in Figs. (2, 3). In order to simplify the problem, we only analyzed the force situation of a player of each team, and the weight of no rope. The $f_{A}$ and $f_{B}$ is static friction force on the ground of athletes, $F_{A}$ is a pull of player $A$ from player $B$, and $F_{B}$ is a contrary direction force. Newton's third law tells us that the object B must at the same time give object A counter-acting force when the object $A$ do the force for object $B$, between the two objects of action and reaction are always equal and opposite direction, and in A straight line. From which we can analyze, player $\mathrm{A}$ must get a reaction $\mathrm{F}_{\mathrm{A} 1}$ from $\mathrm{B}$, and player $\mathrm{B}$ must get reaction $\mathrm{F}_{\mathrm{B} 1}$, and $\mathrm{F}_{\mathrm{A}}=\mathrm{F}_{\mathrm{B} 1}, \mathrm{~F}_{\mathrm{A} 1}=\mathrm{F}_{\mathrm{B}}$.

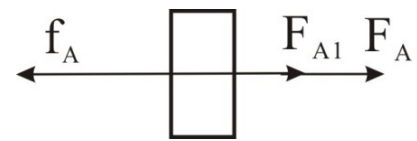

Fig. (2). The force condition of object A.

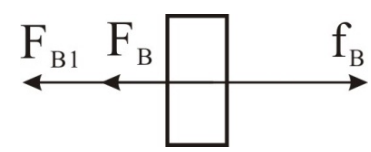

Fig. (3). The force condition of object B.

\section{THE FORCE ANALYSIS OF THE ATHLETES}

From the above, we can see that the athlete move to which direction, depends on the direction of the athletes by force, if the $f_{A}-\left(F_{A}+F_{A 1}\right)>0$, player $A$ win when player $A$ moves the left [9]. If the $f_{B}-\left(F_{B}+F_{B 1}\right)>0$, player $B$ win when player $\mathrm{B}$ moves the right. According to the Newton's third law analysis, we can know, $\mathrm{F}_{\mathrm{A}}+\mathrm{F}_{\mathrm{A} 1}=\mathrm{F}_{\mathrm{B}}+\mathrm{F}_{\mathrm{B} 1}$. Visibly, both players between the pulling force is not the decisive factors, and the main factor that decided the outcome is that athletes and the ground the static friction force between the $f_{A}$ and $f_{B}$, that is to say, the big side of static friction force will win.
So, the size of the static friction force depends on what factors? As can be seen from the formula of friction force $f=$ the $\mu \mathrm{N}$, the size of the friction depends on the size of the coefficient of friction and the positive pressure. The positive pressure in the tug of war, is a complex variable force, it is mainly from the following three aspects.

\subsection{The Athletes' body Weight}

The athletes' body weight directly produces Newton's positive pressure on the ground, so the game should be selected when weight is bigger, the greater the weight, the positive pressure, the greater the friction force is big.

\subsection{The Athlete's Strength}

In the tug of war, we require athletes to backward, foot hard ground at the same time, it will produce a sloping down to the ground pressure $\mathrm{F}$. the force is decomposed into two $\mathrm{F}_{1}$ ' and $\mathrm{F}_{2}$ 'force, we can be seen from the Fig. (4), $\mathrm{F}_{1}{ }^{\prime}$ is the positive pressure on the ground, the force will increase by the friction of the athletes.

\subsection{The Pull of the Other Athletes}

When the athletes toward hypsokinesis, they also can use the pull $\mathrm{F}_{\mathrm{A}}$ of other persons, make their athletes increase positive pressure, we decompose the $\mathrm{F}_{\mathrm{A}}$ for $\mathrm{F}_{\mathrm{A} 1}$ and $\mathrm{F}_{\mathrm{A} 2}$ as shown Fig. (5), $F_{\mathrm{A} 2}$ will make players forward, and $\mathrm{F}_{\mathrm{A} 1}$ will reach the ground through the athlete's body. If the force $F_{A 1}$ from the ground is decomposed according to Fig. (6), apparently, $\mathrm{F}_{\mathrm{A} 1}$ will produce the same effect as the athlete pedal force $F_{1}$, which can increase the athletes on the ground stress [10].

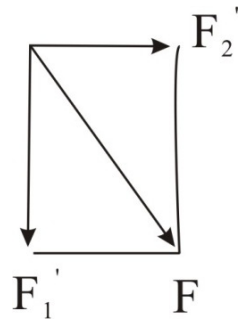

Fig. (4). The decomposition of F.

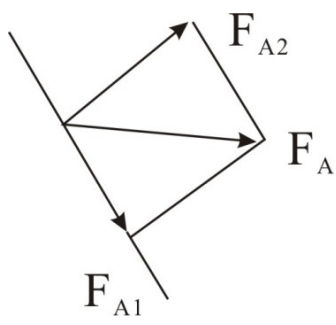

Fig. (5). The decomposition of FA.

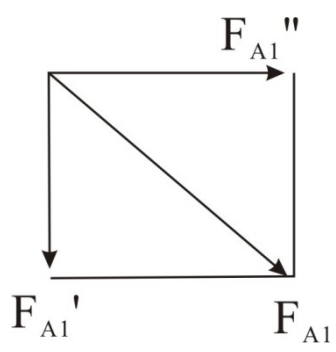

Fig. (6). The decomposition of FA1. 
Through the above analysis, we can obtain the size of the friction force is:

$\mathrm{f}=\mu \times\left(\mathrm{mg}+\mathrm{F}_{1}{ }^{\prime}+\mathrm{F}_{\mathrm{A} 1}{ }^{\prime}\right)$

We can see from the formula, increasing coefficient of friction can increase friction, so the game players wear shoes sole with concave and convex decorative pattern, in order to increase the coefficient of friction, the friction force increases. In terms of positive pressure, in addition to the athletes' body weight, $\mathrm{F}_{1}$ and $\mathrm{F}_{\mathrm{Al}}$ plays a very important role, and that the size of the two forces in addition to the size of the athlete's strength, still depends on the athletes skill, also is the angle of the backward athletes, if back angle is moderate, will produce very good result [11]. The team athletes overall cooperation is also very important. If all the athletes in a short time put forth their strength and in a short time the resultant force is greater than the dispersion force, the friction will be bigger, make the other side difficult to pull own athletes, which lays a foundation for success.

\subsection{The Analysis of Skill}

In a tug of war, pull each other is basically in a horizontal direction, a center of gravity of body up quickly, basically not affected by each other. As long as their original body posture is correct, low center of gravity, the body get up instantaneous acceleration is not a problem. At the same time, it produces upward acceleration, feet have larger frictional force, as long as the friction are greater than the other side between the friction and ground, back in the center of gravity of the body will become a reality. A ground, stand up, after the completion of the action. Under the condition of invariable in a foothold, center of gravity has been back, it's more heavy torque. Then the center of gravity is taken lower, properly change the foothold, prepared for the next hard ground, rise up quickly [12].

The size of hand grip has some influence on the outcome of the tug of war. Hands and the rope there should be no relative sliding. It's important for the hold the rope pressure. Set the static friction coefficient is $\mu_{0}$ between the hand and rope, the maximum pressure when holding the rope to the rope is $\mathrm{N}_{\max }$, and then the maximum static friction force is $\mu_{0} \mathrm{~N}_{\text {max }}$ between the rope and the hand. When the other side of the rope pulling force is greater than his own hand and the rope between the maximum static friction force when the hand with sliding occurs between the rope, the static friction force of sliding friction force and the maximum, so the other party, together with the rope to the rear of sports have been unable to stop, failure is not saved. In ground movement, is mainly completed by lower limb muscle coordination, and the size of the grip strength mainly related to the degree of upper limb muscles. If the players do ground movement, the hand between the rope and sliding, ground movement is in- valid. This requires powerful tug-of-war team limbs should be strong.

\section{CONCLUSION}

Within the scope of the whole world, tug-of-war is profound origin early and is most common and most ancient folk custom sports. Tug-of-war posture should be changed correspondingly according to the different situation of the game. Through the above analysis we can see that applying force to the other athletes is a very key factor, especially athlete pedal soil $\mathrm{F}$ on the surface, the force of reaction will enable athletes move into the opposite direction. On the premise of enough friction, under the action of soil fertility $\mathrm{F}$, athletes can pull the other athletes. Weight is the important factors that affect the tug of war competition. From the above analysis, when a certain the friction factor, the greater the weight and the maximum static friction force is, the greater the more advantageous against up. At the same time, every athlete applying skills and work time is one of the important factors to success.

\section{CONFLICT OF INTEREST}

The author confirms that this article content has no conflict of interest.

\section{ACKNOWLEDGEMENTS}

Declared none.

\section{REFERENCES}

[1] Q. Li, The Course of Sports Technology, Harbin Industrial University Press, Harbin, 2002.

[2] Q. A. Shen, and C. Du, Mechanics, Higher Education Press, Beijing, 2002.

[3] K. Liu, Physics, Higher Education Press, Beijing, 2002.

[4] X. Liu, History of China, Economic Daily Press, Beijing, vol. 127, 2001.

[5] S. Liu, "Ten thousand people in the tug-of-war ancient and modern talk about," Modern Sports Weekly, vol. 20, no. 6, pp. 33-36, 2007.

[6] J. Li, "Tug-of-war sport new test," Journal of Sport Culture Tribune, vol. 15 , no. 5 , pp. $92,2002$.

[7] National Research Council, "A Framework for K-12 Science Education: Practices, Crosscutting Concepts, and core Ideas," The National Academies Press, Washington, D. C, 2011.

[8] S. Wei, and Y. Jia, "Learn the advanced: to promote curriculum, teaching and evaluation Consistency," Journal of Global Education Prospects, no. 9, pp. 24-31, 2010.

[9] W. Liu, and J. Ruan, "The origin, development and winning techniques of tug of war," Sports Adult Education Journal, vol. 20, no. 3, 2004.

[10] P. Shen, "The mechanics analysis of tug-of-war," Physics Teachers, vol. 31, no. 10, 2010.

[11] B. Liu, and X. Du, "The biomechanics analysis of tug-of-war technology," Journal of Nanjing Institute of Sport (Natural Science Edition), vol. 2, no. 3, 2003.

[12] S. Yan, Beijing, "Athletic Sports People's Sport Publishing House," 2006. 